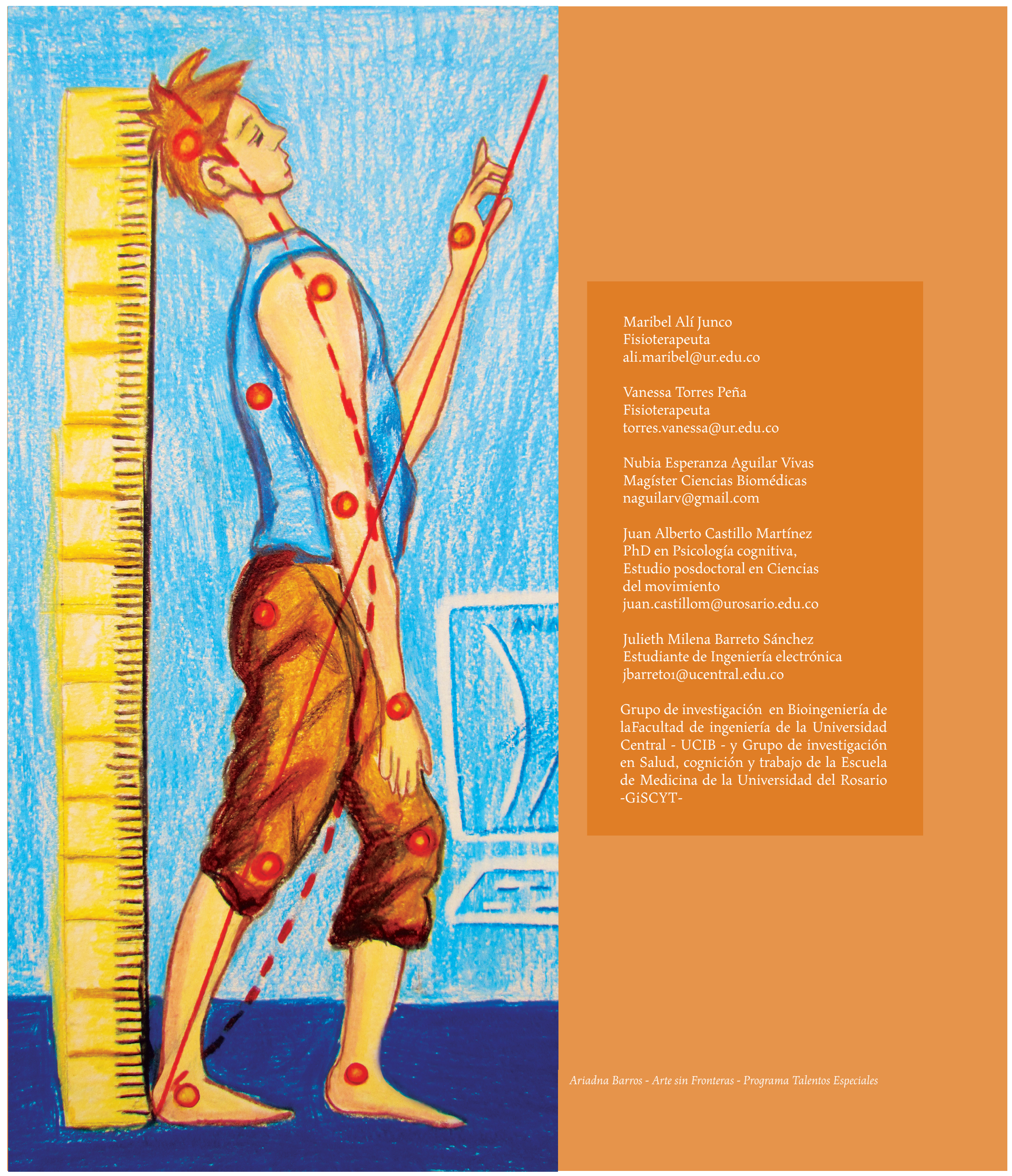




\title{
APLICACIONES TECNOLÓGICAS EN ANÁLISIS DE MOVIMIENTO: DIAGNÓSTICO Y SEGUIMIENTO PARA PROCESOS FISIOTERAPÉUTICOS
}

\author{
Technological Applications in Motion Analysis: Diagnosis \\ and Monitoring for Physiotherapy Process
}

Fecha de Recepción: 17 de Febrero de 2011 - Fecha de Aprobación: 16 de Agosto de 2011

\begin{abstract}
RESUMEN
El presente trabajo muestra las perspectivas de aplicación y uso de la tecnología de análisis de movimiento para el diagnóstico y seguimiento en procesos terapéuticos, mediante el análisis de parámetros de movimiento, apoyado en la tecnología de BTS SMART-E. Esta tecnología permite el análisis de la cinemática de los movimientos corporales y registra datos e imágenes en tres dimensiones (3D), para permitir en análisis y la valoración del movimiento en los planos frontal, sagital y trasversal. El objetivo de este estudio es mostrar las posibles aplicaciones y el uso de esta tecnología. Se presentan los modelos para tres estudios de aplicación realizados: balance corporal, comportamiento de mano luego de una enfermedad cerebro vascular (ECV) y análisis de inestabilidad en columna lumbar. Estos modelos muestran la posibilidad de medir el comportamiento de uno o varios ángulos, en cuanto al desplazamiento, la velocidad y la aceleración. Este tipo de tecnología permite objetivar la evaluación y garantiza la reproducción de las pruebas en diferentes escenarios.
\end{abstract}

PALABRAS CLAVE

Análisis de movimiento, biomecánica, fisioterapia, diagnóstico, tecnología

\section{ABSTRACT}

This work shows the perspectives for application and use of motion analysis technology in diagnosis and monitoring therapeutic process, through the analysis of motion parameters, supported by technology BTS SMART-E. This technology allows the analysis of the kinematics of body movement and capture data and images in three dimensions $(3 \mathrm{D})$. The aim is to enable analysis and assessment of movement in the frontal, sagittal and transversal plane. The interest of this study is to show the possible applications and the use of this technology. For three studies, the models are presented: body balance, hand behavior after stroke and analysis of lumbar spine instability. These models show the possibility of measuring the behavior of one or more angles, in terms of displacement, velocity and acceleration. This technology allows objectifying the evaluation and ensuring the reproduction of the tests in different scenarios

KEY WORDS

Motion analysis, biomechanics, physical therapy, diagnosis, technology 
El primer sistema fue desarrollado con cámaras-TV para registrar y analizar el movimiento de varios marcadores en tiempo real, se llevo a cabo en Delft-Holanda (1967). Sobre este sistema se ha basado la Universidad de Strathclyde en el Reino Unido, para la creación del primer sistema comercial conocido como VICON (1980) antecesor del VICON-VX (1989). Más adelante (1986), aparecen sistemas como ELITE y ExpertVision. Hoy en día, los sistemas optoelectrónicos basados en cámaras-TV se siguen utilizando pero con mejoras notables que tienen mayor calidad y ahorro de tiempo.

El sistema ELITE (Elaboratore di Immagini Televisive): fue desarrollado en primera instancia por el Centro de Bioingeniería de Milán (1983). Con este sistema se pueden realizar análisis cinemáti$\cos 2-\mathrm{D}$ a $50 \mathrm{~Hz}$ o 3-D a $100 \mathrm{~Hz}$ en condiciones de laboratorio o en el campo (13).

El sistema VICON VX (Video Converter for Biomechanic): se ha introducido con gran éxito desde (1989), gracias a su exactitud en las coordenadas espaciales de los marcadores y la posibilidad de disponer de las trayectorias tridimensionales de éstos al poco tiempo de haberse realizado la toma. $(13,14)$. El sistema PRIMAS (Precision Motion Analysis System): permite obtener datos con menos "ruido" y supervisa la evolución de los ensayos o genera feedback en tiempo real (15). El sistema ExpertVision HiRes 3D (High Resolution 3D Motion Analysis System): es un sistema completo en tiempo real en 2-D y 3-D. Posee características importantes como el tratamiento conjunto de los datos cinemáticos, cinéticos y electromiográficos (EMG) $(16,17)$.

Las tecnologías que son usadas en la actualidad a nivel mundial incluyen dispositivos opto-electrónicos para el análisis biomecánico, como los llamados laboratorios de análisis de movimiento o análisis de marcha; uno de estos sistemas es el BTS SMART-E que puede medir en cualquier tipo de movimiento corporal, el desplazamiento angular, velocidad y aceleración, con relación al tiempo. En estudios de análisis de movimiento humano se utilizan diferentes equipos; BTS SMART-D, SMART (BTS. Milan, Italy) y Vicon MX, que son sistemas digitales optoelectrónicos comunes y de alta precisión para el análisis del movimiento. Estos sistemas consisten básicamente en un conjunto de cámaras infrarrojas directamente conectadas a una caja de integración que contiene el software adecuado para la captura de datos, y reconstrucción $3 \mathrm{D}$ de los movimientos humanos $(2,3,4,5,6)$. Esta tecnología permite ampliar el campo de la investigación de la cinemática a través de un software que crea una visión tridimensional y obtiene datos de variables como ángulos, velocidad y aceleración de determinado movimiento en tiempo real. También permite de manera rápida, objetiva, precisa y no invasiva el estudio del movimiento humano y un análisis computarizado de este, aplicado al campo medico y fisioterapéutico; lo que establece un diagnóstico y tratamiento preciso en el manejo de una patología o en el estudio de variaciones anatómicas dadas por las diferentes articulaciones $(7,8)$.

El objetivo del análisis del movimiento es medir cuantitativamente los esquemas o modelos de movimiento del ser humano, identificar alteraciones y observar la postura con el fin de generar posibles tratamientos $(9,10,11)$. Para lograr este objetivo se han desarrollado diversas tecnologías de registro, las cuales se presentan a continuación (12).

\section{Sistemas basados en sensores PSD (Position-sensitive devices).}

Su característica más importante es el sensor o foto detector PSD cuyo principio de funcionamiento se conoce como "foto-efecto lateral". A partir de la información conseguida por dos cámaras, el sistema establece la posición espacial del punto luminoso. El sistema SELSPOT II (Selective Light SPOT recognition): su aplicación es biomédica y últimamente está incorporado el programa MULTI-Lab para el tratamiento de datos cinemáticos simultáneamente con datos de otros sistemas de medida. Como desventaja se encuentra que el cableado en el sujeto genera incomodidad y en el caso de registrar al mismo tiempo EMG es posible que se den interferencias entre las señales. Como ventajas se tienen la identificación automática de los marcadores y la buena resolución espacio-temporal.

\section{Sistemas basados en sensores CCD de exploración de línea}

Los sistemas de esta familia utilizan emisores de luz infrarroja (IRLEDS) como marcadores, y su componente básico es un sensor CCD de exploración de línea. El sistema OPTOTRAK: desarrollado por Northern Digital, está basado en sensores de exploración de línea (1-D) y puede configurarse según los objetivos de la aplicación.

\section{Sistemas basados en scáners mecano-ópticos}

El sistema CODA (Cartesian Optoelectronic Dynamic Anthropometer): desarrollado en Loughborough en Reino Unido (1981) ha evolucionado en el sistema CODA MPX30 (1988). Esta tecnología 
(Mitchelson, 1988) utiliza tres escáners rotatorios, dos giran respecto a un eje vertical y el otro (intermedio) a un eje horizontal. Los dos escáners exteriores emiten cada uno un haz de luz coloreada (azul - rojo - infrarrojo). Este sistema tiene como ventaja el uso de marcadores sin cableado (13).

Los equipos como VICON, ELITE, y Max Reflex son usados por ejemplo en la valoración de la marcha, en donde se realiza una reconstrucción en $3 \mathrm{D}$ de los marcadores logrando crear un algoritmo aplicable a la realización de una tarea, lo que genera una amplia gama de aplicaciones para el análisis de movimiento. (18, 19, 2o). Otros importantes sistemas de análisis como: El sistema tridimensional Zebris CMS 7oP, CA 6000 es utilizado para determinar el rango de movimiento de la columna lumbar en población turca para proveer un diagnostico de los problemas de la columna y también tener un seguimiento del tratamiento. Este sistema detecta y registra los cambios que ocurren en el movimiento angular de la articulación mediante el uso de ondas sonoras de alta frecuencia (21). Otros como una plataforma de fuerza dirigida por un sistema Pedar, permite analizar el mecanismo de adaptación neuromuscular que utilizan los miembros inferiores en la marcha, cuando se deslizan sobre superficies resbaladizas, estos son evaluados en el programa Pascal por Windows 1.5; Borland International Inc, Austin, TX (22).

La captura optoelectrónica del sistema VICON 250 3D se utilizó para investigar los patrones angulares de las articulaciones metacarpofalángicas de los dedos 1-5 durante el agarre y se ven afectadas por patologías como la artritis o diferentes traumas. Se utilizaron 5 cámaras estroboscópicas, que colectan información a una frecuencia de $120 \mathrm{~Hz}, 26$ marcadores reflectivos con un diámetro de $5 \mathrm{~mm}$, y un computador con un software específico $(23,24)$. Un sistema Vicon MX (Oxford Metrics Ltd, Oxford, England) con 10 cámaras $\left(8 \times \mathrm{MX}_{3}\right.$ and $2 \times \mathrm{MX}_{40}$ ) operando a una frecuencia de $100 \mathrm{~Hz}$ fue utilizado para evaluar el síndrome de dolor patelofemoral y evaluar la postura del pie para el uso de ortesis $(25,26,27)$. Otro estudio fue realizado con un sistema Vicon 612 y Vicon Nexus 1.4 Data. (Vicon Motion Systems Ltd., Oxford, UK) permitió analizar la cinemática del pie a una frecuencia de $100 \mathrm{~Hz}$, utilizando un modelo validado para adultos sanos, luego adaptado para niños en donde se valoro el ciclo de la marcha $(28,29)$. El sistema de análisis de movimiento con vista 3D (Motion Analysis Corporation, Santa Rosa, CA), se utilizó para la medición angular de los dedos, obteniendo datos comparables con los del goniómetro convencional (30), Otro estudio lo utiliza para determinar el movimiento de las tres articulaciones del pulgar durante la ejecución de actividades de la vida diaria, lo cual proporciona una referencia para médicos y terapeutas en la evaluación e intervención en pacientes con deficiencias del pulgar (31). Un sistema Vicon, Oxford, UK; Model 46o permitió evaluar parámetros tradicionales como las asimetrías o simetrías durante el ciclo de la marcha; este utiliza el laboratorio de análisis de movimiento con un aparato ortopédico o corsé que permitió cuantificar las asimetrías e identificar las patologías o lesiones asociadas, para la creación de tratamientos específicos $(32,33,34)$. Por medio del sistema tridimensional de análisis de movimiento (CODA System.6, Charnwood Dynamics, Ltd, UK) se registraron datos cinemáticos para el análisis de las extremidades inferiores durante el ciclo de la marcha, en sujetos con síndrome de cordón central (CCS); los cuales ayudaron a comprender cómo afecta este en la marcha y a diseñar las estrate- gias de rehabilitación para su tratamiento (35). También el sistema VICON 370; fue útil para realizar la caracterización cuantitativa de la cinemática del pie $(36,37)$. Todos estos estudios muestran el uso de diferentes sistemas tecnológicos que pueden ser usados para el diagnostico y seguimiento de diversas patologías a través de un análisis biomecánico.

\section{METOdología}

La metodología empleada en este trabajo incluye, los sistemas tecnológicos asociados al laboratorio de análisis de movimiento BTS SMART-E, y su aplicación práctica para el análisis de variables en tres casos de estudio: balance corporal, comportamiento de mano luego de un ECV y análisis de inestabilidad en columna lumbar.

Para el análisis de movimiento se cuenta con un sistema BTS SMART-E, este moderno sistema permite el análisis y el registro completo de los parámetros cinemáticos del cuerpo durante cada movimiento; consta de seis cámaras optoelectrónicas de luz infrarroja (IRLEDS) de alta resolución, una plataforma de fuerza, marcadores reflectivos, marco de referencia ejes X,Y y Z (figura 1) y la integración de un software que incluye tres programas: Smart Capture, que permite realizar el proceso de calibración de las cámaras y el registro de captura de datos, Smart Tracker realiza el proceso de creación del modelo anatómico (figuras 2,3 y 4) y Smart Analyzer es usado para realizar un completo análisis del gesto motor en tres dimensiones $3 \mathrm{D}$.

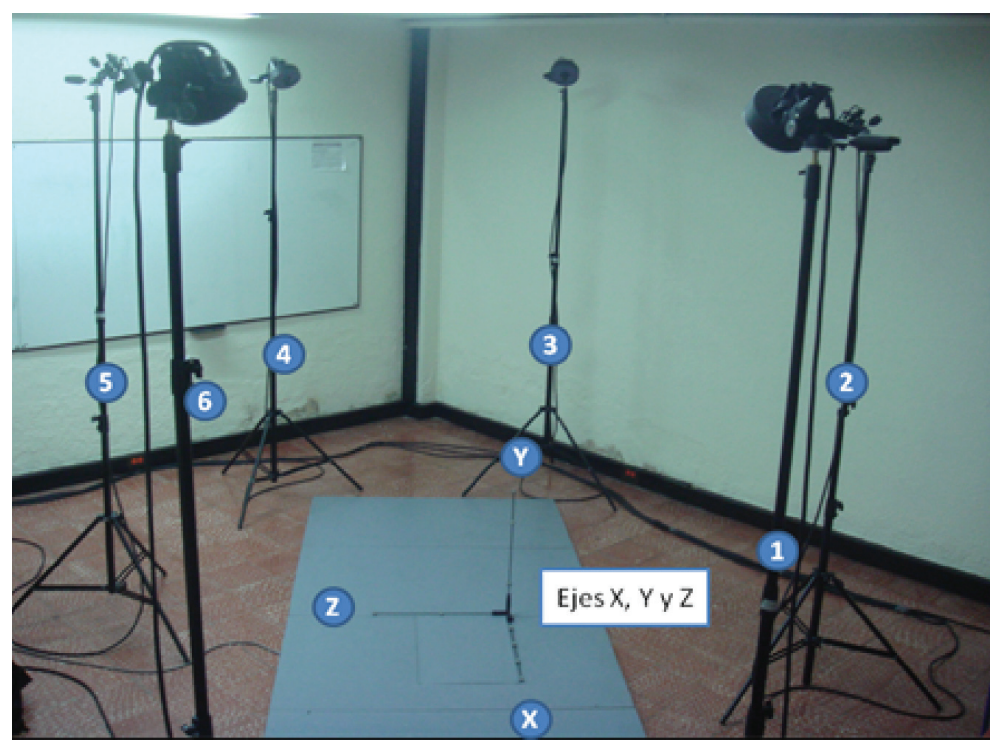

Figura 1. Disposición de las cámaras para iniciar el proceso de calibración y ubicación de los ejes con respecto a la plataforma.

Después de calibrar el sistema, se procede a la captura de los movimientos corporales en cada prueba o caso de estudio. Para los casos de inestabilidad de columna lumbar y balance corporal, se tuvo como referencia el protocolo de Davis (38) con modificaciones de acuerdo a cada análisis, en el caso de comportamiento de mano luego de un ECV, se diseño un modelo como propuesta inicial de estudio (Figuras 1, 2,3 y 6). 
En la captura realizada para el análisis de inestabilidad de columna lumbar, se registraron movimientos de flexión-extensión e inclinación lateral izquierda y derecha de tronco; con previa colocación de los marcadores reflectivos (figura 1, 5). Adicionalmente en este estudio, se usó tecnología de análisis electromiográfico (EMG), en los músculos multifidos, oblicuos internos y externos y transversos.



Figura 2. Modelo utilizado para análisis de inestabilidad de columna lumbar; los marcadores son: ACRDER (Acromion derecho), ACRIZQ (Acromion Izquierdo), T12 (ultima vertebra torácica), L4 (cuarta vértebra lumbar), EIASD (espina iliaca antero-superior derecha), EIASIZ (espina iliaca antero superior izquierda).

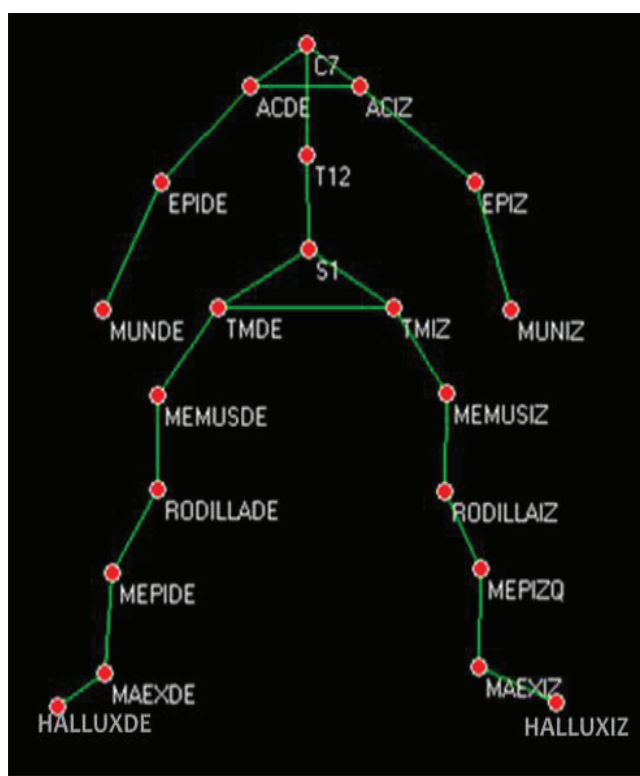

Figura 3. Modelo utilizado para análisis de balance corporal. ACDE-ACIZ (Acromion derecho e izquierdo),EPIDE-EPIZ(Epicondilo derecho e izquierdo), MUNDE-MUNIZ (Apófisis estiloides del radio derecha e izquierda), C7 (séptima vértebra cervical), $T_{12}$ (doceava vertebra torácica) $S_{1}$ (primera vertebra sacra), TMDE-TMIZ (Trocánter mayor derecho e izquierdo), MEDUSDE-MEDUSIZ (mitad de muslo derecho e izquierdo), RODILLADE-RODILLAIZ (cónditos femorales derecho e izquierdo), MEPIDE-MEPIZQ(mitad de pierna derecha e izquierda), MAEXDE-MAEXIZ(maléolos externos derecho e izquierdo), HALLUXDE- HALLUXIZ (base del primer metatarsiano derecho e izquierdo).

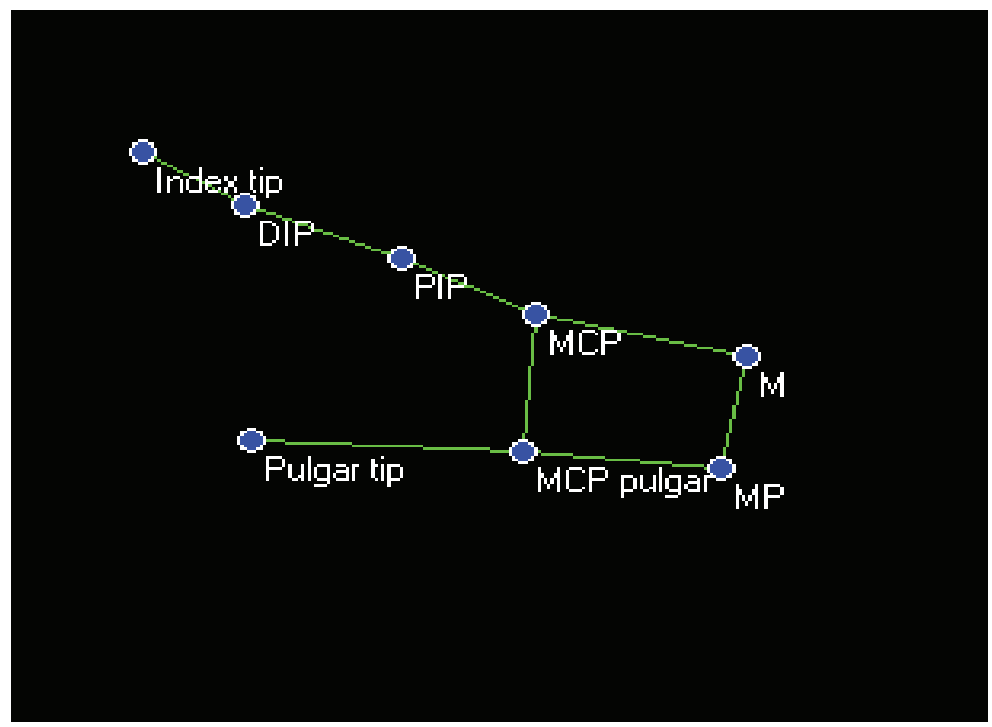

Figura 4. Modelo utilizado para análisis de mano luego de un ECV Pulpejo (index tip), interfalangica distal (DIP), interfalangica proximal (PIP), metacarpofalángica (MCP), base del 2do metacarpiano(M), base del 1er metacarpiano (MP), metacarpofalángica del pulgar (MCP pulgar), pulpejo del pulgar (pulgar tip).

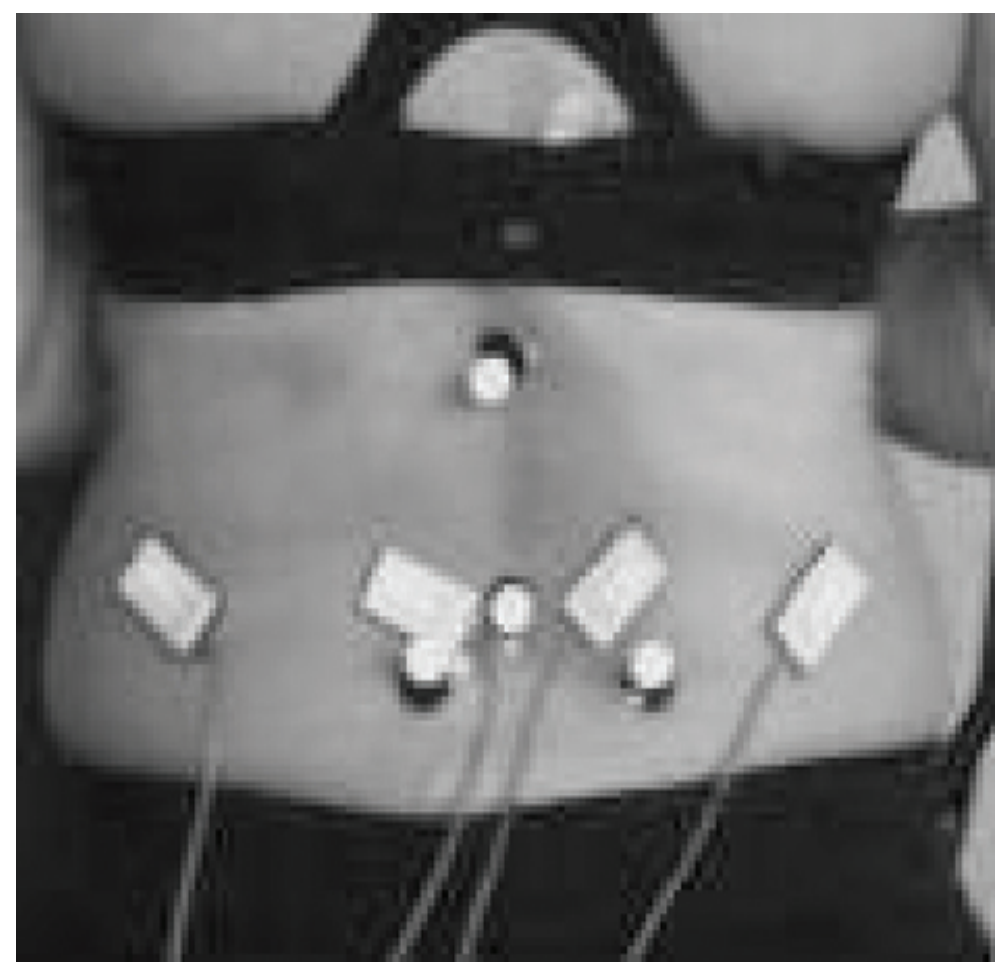

Figura 5. Paciente con marcadores y sensores de EMG para el análisis de inestabilidad de columna.

En el caso de balance corporal se registran los movimientos incluidos en el Test de Tinetti (39), con ubicación de marcadores (figura 6) y en el caso de análisis de mano luego de un ECV se realizan movimientos de aproximación y agarre de objetos, con ubicación de marcadores (figura 7). 


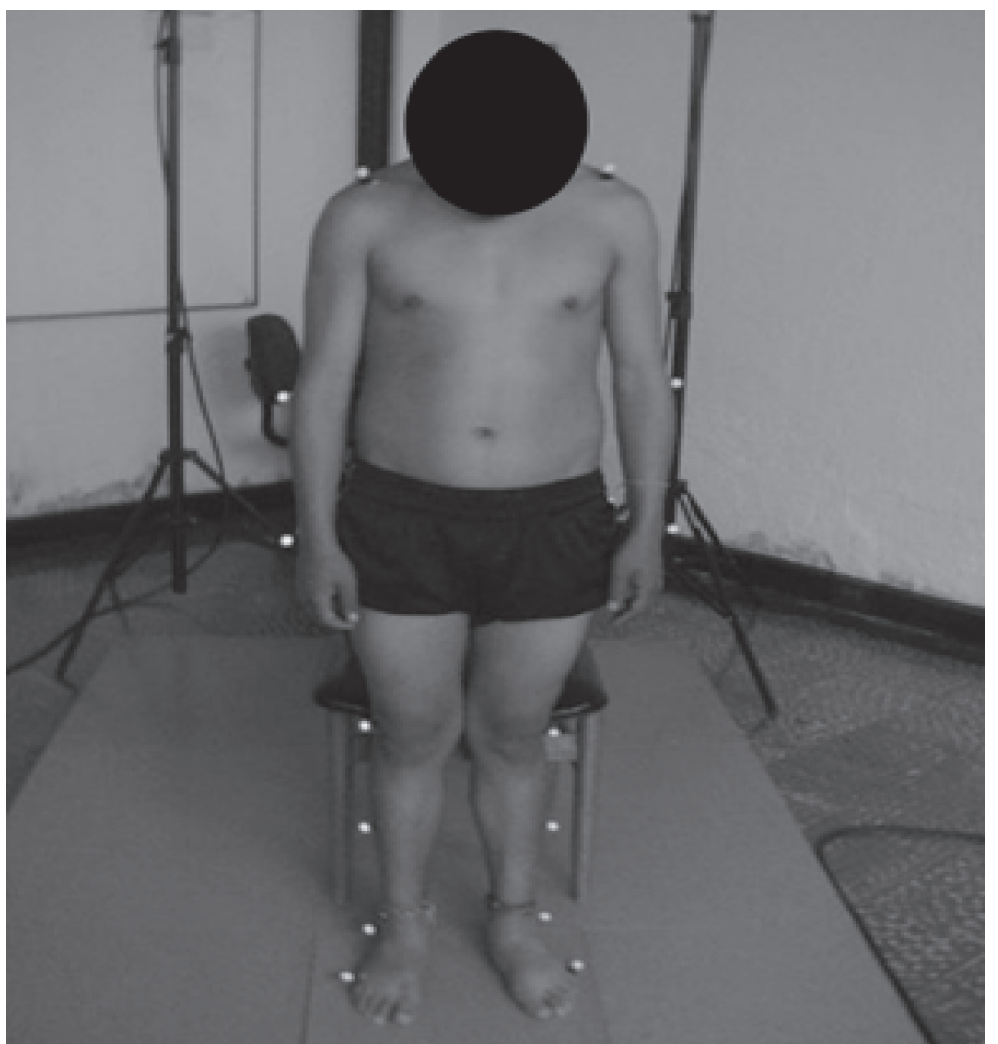

Figura 6. Paciente con marcadores según protocolo de Davis.

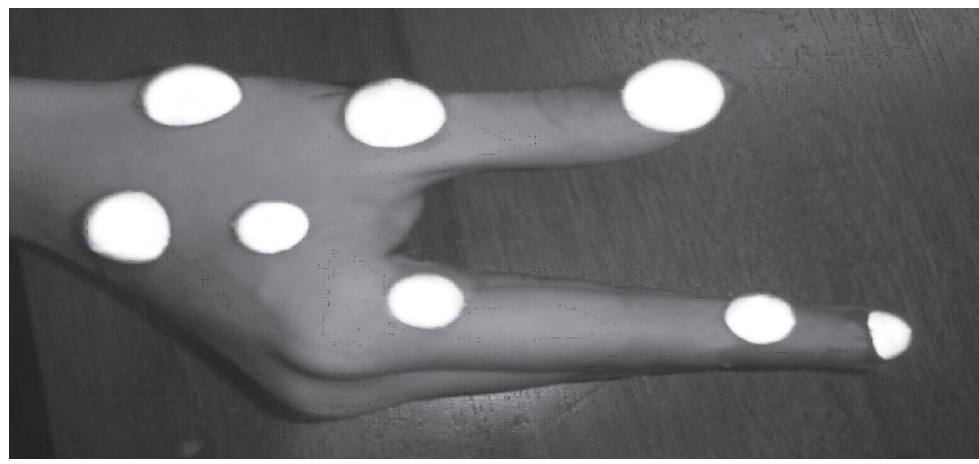

Figura 7. Paciente con marcadores para análisis de mano luego de un ECV.

Con esta metodología se pueden obtener: videos 3D de cada paciente, en cada una de las pruebas que realice; registros gráficos y numéricos del comportamiento motor cada 0.02 segundos, lo que permite realizar un análisis detallado y cuantitativo del movimiento que se desee evaluar, y por ende no solo diagnosticar claramente la patología en cuestión, sino también hacer un seguimiento cronológico de los procesos de rehabilitación.

\section{RESULTADOS}

Los resultados arrojados por el sistema de análisis de movimiento BTS SMART-E son: vista 3D en tiempo real (figura 8) para el estudio del valor de desplazamiento angular en grados, los ángulos a estudiar los determina cada profesional de acuerdo a la variable de estudio de su interés, por ejemplo la medida especifica del ángulo para balance corporal (Figura 9). Adicionalmente se obtienen tablas


Figura 8. Vista tridimensional en Smart Capture. A, balance corporal. B. comportamiento de mano luego de un ECV. C. inestabilidad de columna. 
organizadas con datos numéricos del movimiento realizado, en los lapsos de tiempo que se deseen analizar (Tabla 1), sabiendo que el sistema permite tomarlos cada 0.02 segundos.

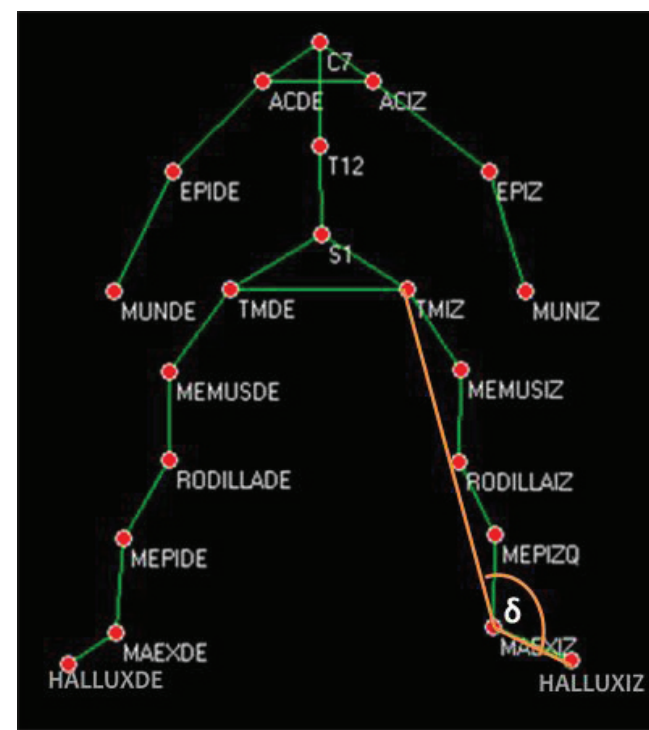

Figura 9. Muestra la formación del ángulo.

Entre los puntos TMIZ (Trocánter mayor izquierdo), MAEXIZ (maléolo externo izquierdo) HALLUXIZ (base del primer metatarsiano izquierdo).

Se obtienen gráficos de cada estudio y variables como: desplazamiento, velocidad y aceleración angular (figura 10). Con los diferentes datos obtenidos, se pueden evidenciar las características individuales de cada paciente, durante un movimiento o prueba específica y así valorar su condición estructural $y / o$ funcional.

\section{DISCUSIÓN}

La utilización de sistemas de análisis de movimiento se convierte en una herramienta de gran utilidad en el campo de investigación dentro del área de fisioterapia y otras ciencias de la salud $(13,14,15,16,17)$. Este trabajo busca incentivar al profesional de la salud, a utilizar sistemas tecnológicos de última generación, permitiéndole cuantificar y mejorar sus procesos de análisis y evaluación fisioterapéutica.

Cuando observamos los procesos desarrollados para analizar la inestabilidad de columna lumbar nos damos cuenta que en su mayoría son de tipo cualitativo, como por ejemplo test $\mathrm{H}$ y test I (40). Estos procesos requieren una ayuda tecnológica como la propuesta en este trabajo, con el diseño inicial de un modelo (colocación estratégica de los marcadores), la captura de los movimientos específicos y el estudio cuantitativo de desplazamientos del tronco. En el caso de balance corporal existen diversas pruebas también de carácter cualitativo tales como: El test Get up and go, Test Tinetti, Evaluación cronometrada de la estación unipodal, Prueba de alcance funcional, Test de Romberg, Prueba de Romberg mejorada, entre otros (41). También requieren de tecnología para medir el desplazamiento del centro de gravedad del cuerpo, el comportamiento de los ángulos generados por los referentes anatómicos en las posiciones sedente y bípeda y la velocidad y aceleración del desplazamiento $360^{\circ}$ (39). En el estudio del comportamiento de mano durante el agarre en pacientes después de ECV, se pudo generar una propuesta de modelo de estudio que permitió cuantificar el desplazamiento de los ángulos de las diferentes articulaciones de la mano, la velocidad de agarre y aproximación a un objeto. Por ejemplo en este caso se tuvo la posibilidad de la aplicación y seguimiento de un tratamiento que pretendía mejorar la funcionalidad de la mano y juzgar en el tiempo su efectividad.

\begin{tabular}{|c|c|c|c|}
\hline TIEMPO & $\begin{array}{c}\text { ANGULO ENTRE } \\
\text { T12, I4, EIPSD PARA } \\
\text { INESTABILIDAD DE } \\
\text { COLUMNA }\end{array}$ & $\begin{array}{l}\text { ANGULO } \\
\text { EQUILIBRIO } \\
\text { TINETTI }\end{array}$ & $\begin{array}{l}\text { ANGULO } \\
\text { MANO } \\
\text { (MCP), (M), } \\
\text { (MP), }\end{array}$ \\
\hline 0.460 & 127.515 & 112.053 & 63.936 \\
\hline 0.480 & 127.623 & 112.105 & 63.941 \\
\hline 0.500 & 126.665 & 112.144 & 63.973 \\
\hline 0.520 & 124.549 & 112.210 & 63.992 \\
\hline 0.540 & 124.360 & 112.128 & 64.028 \\
\hline 0.560 & 124.414 & 112.085 & 64.043 \\
\hline 0.580 & 124.468 & 111.895 & 64.010 \\
\hline 0.600 & 124.195 & 111.756 & 64.014 \\
\hline 0.620 & 124.194 & 111.701 & 63.993 \\
\hline 0.640 & 124.805 & 111.675 & 63.881 \\
\hline 0.660 & 126.075 & 111.644 & 63.885 \\
\hline 0.680 & 127.489 & 111.792 & 64.018 \\
\hline 0.700 & 127.903 & 111.711 & 64.024 \\
\hline 0.720 & 127.755 & 111.572 & 63.911 \\
\hline 0.740 & 126.188 & 111.674 & 63.956 \\
\hline 0.760 & 124.688 & 111.854 & 64.107 \\
\hline 0.780 & 125.520 & 111.933 & 64.134 \\
\hline 0.800 & 125.895 & 111.967 & 64.048 \\
\hline 0.820 & 125.926 & 112.069 & 64.072 \\
\hline 0.840 & 126.030 & 112.166 & 64.005 \\
\hline 0.860 & 127.009 & 112.087 & 63.932 \\
\hline $\begin{array}{c}\text { CAMBIO } \\
\text { DESPLAZAMIENTO } \\
\text { ANGULAR }\end{array}$ & 0.506 & -0.034 & 0.004 \\
\hline
\end{tabular}

Tabla 1. Desplazamiento de cambio angular en los diferentes modelos.

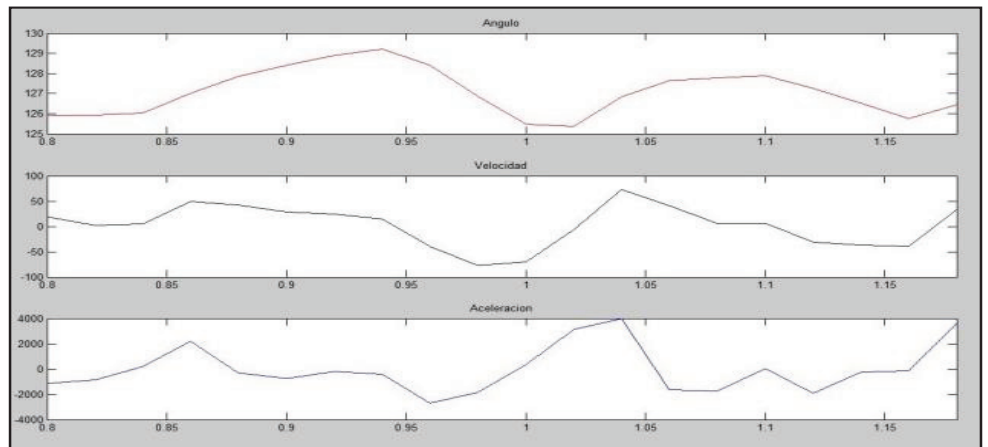

Figura 10. Grafica del desplazamiento angular, velocidad y aceleración entre los puntos T12, L4, EIPSD, con vértice del ángulo en L4, en donde se observa el comportamiento en el tiempo, en análisis de inestabilidad de columna lumbar. 
$\mathrm{Al}$ poder cuantificar todos estos procesos, se abre la posibilidad de comparar datos obtenidos en el caso patológico de estudio, con población control evaluada con la misma metodología y así llevar a cabo investigaciones de carácter comparativo, altamente confiables y reproducibles.

\section{CONCLUSIONES}

El uso de un laboratorio de análisis de movimiento. Se constituye en una herramienta de gran utilidad en el campo de la determinación de estrategias terapéuticas, de técnicas de rehabilitación y en general para la investigación fisioterapéutica. Los diagnósticos, evaluaciones y estudios realizados utilizando esta metodología, permiten desarrollar protocolos asociados a las diferentes patologías que dan la posibilidad de cuantificar los procesos de evaluación y de esta manera estandarizar los procedimientos y metodologías de estudio.

Es importante considerar que el uso de las tecnologías de análisis de movimiento, garantiza la reproductibilidad de cada una de las evaluaciones diagnósticas que se requieran. La protocolización de un proceso evaluativo permite establecer cómo se debe medir las variables requeridas para la elaboración de un diagnostico y a partir de allí hacerlo repetible de la misma forma a un paciente, utilizándolo para establecer la evolución y aplicándolo antes, durante y después de un tratamiento. El trabajo realizado es base para la propuesta de diferentes estudios de investigación formal, en el área de fisioterapia.

\section{AGRADECIMIENTOS}

A la Universidad Central por permitirnos el uso del laboratorio de análisis de movimiento BTS SMART-E. A la Universidad del Rosario por su apoyo durante el proceso de estudio y a los investigadores Jonathon O'Brien, María Constanza Trillos y Erika Lozano por sus aportes de datos en casos específicos de estudio.

\section{Referencia:}

Las referencias a otras obras son una parte muy importante en la literatura científica;

\section{ya que estas permiten conocer más sobre los autores y mantener vivas sus voces dentro del texto.}

1. Daza J. Evaluación clínico-funcional del movimiento corporal humano. Bogotá, D.C. Colombia: Médica Panamericana. 2007.

2. Pérez R, Costa U, Torrent M, Solana J, Opisso E, Cáceres C, et al. Upper Limb Portable Motion Analysis System Based on Inertial Technology for Neurorehabilitation Purposes. Sensors 2010; 10: 1073310751.

3. Cerveri P, De momi E, Marchente M, Lopomo N, Baud-bovy G, Barros R. M. L, Ferrigno G. In Vivo Validation of a Realistic Kinematic Model for the Trapezio-Metacarpal Joint Using an Optoelectronic System. Annals of Biomedical Engineering 2008; 36: 1268-1280.

4. Stagni R, Fantozzi S, Cappello A, Leardini A. Quantification of soft tissue artefact in motion analysis by combining $3 \mathrm{D}$ fluoroscopy and stereophotogrammetry: a study on two subjects. Clinical Biomechanics 2005; 20: 320-329.
Sutherland D.H. The evolution of clinical gait analysis part III - kinetics and energy assessment. Gait \& Posture 2005; 21: 447-461.

6. Anderst W, Baillargeon E, Donaldson W, Lee J, Kang J, Validation of a Noninvasive Technique to Precisely Measure In Vivo Three-Dimensional Cervical Spine Movement. Spinejournal 2011; 36: 393-400.

7. Mündermann L, Corazza S, Andriacchi T. The evolution of methods for the capture of human movement leading to markerless motion capture for biomechanical applications. Journal of NeuroEngineering and Rehabilitation 2006, 3:6.

8. Simon S. Quantification of human motion: gait analysis-benefits and limitations to its application to clinical problems. Journal of Biomechanics 2004; 37: 1869-1880.

9. Adams D, Cerney M. Quantifying biomechanical motion using Procrustes motion analysis. Journal of Biomechanics 2007; 40: 437-444. 
10. Chang F, Rhodes J, Flynn K, Carollo J. The Role of Gait Analysis in Treating Gait Abnormalities in Cerebral Palsy. Orthop Clin N Am 2010; 41: 489-506.

11. Cowan s, Bennell k, Crossley k, Hodges p, Mcconnell j. Physical therapy alters recruitment of the vasti in patellofemoral pain syndrome. Medicine \& Science In Sports \& Exercise. 2002; 3412-1879.

12. Rusaw D. Ramstrand N. Motion-analysis studies of transtibial prosthesis users: a systematic review. Prosthetics and Orthotics International 2011; 35: 8-19.

13. Gianikellis, K. Características técnicas y prestaciones de los sistemas optoelectrónicos más difundidos en el campo de la biomecánica del movimiento humano. Estado actual de conocimientos. Revista Motricidad 1996; 2, 191-210.

14. Cramp E, Manual of Motion Lab Systems Inc. 2009. August 19.

15. Jobbágy Á, Furnée E.H, Gyöngy L, Monos E, Harcos P, Martin F. Biomedical Applications Of A Precision Motion Analysis System. Technical University of Budapest, Dept. Measurement and Instrument Engineering. 1994. URL: http://home.mit.bme.hu/ jobbagy/publ/ mechatro7.htm. Cconsultado: junio 10/2011.

16. lan-yuen guo, fong-chin su, chich-haung yang, shu-hui wang, jyh-jong chang, wen-lan wu, et al. Effects Of Speed And Incline On Lower Extremity Kinematics During Treadmill Jogging In Healthy Subjects. Biomedical engineering-applications 2006; 18.

17. Sheng-che lin, tsan-hsun huang, fong-chin su, you -li chou. Motion Analysis Of Mouth Movement Utilizing Challis Technique-Experiment Model And Clinical Study Using Video-Based System. Biomedical engineering-applications $2002 ; 14$.

18. Figueroa P, Leite N, Barros R. A flexible software for tracking of markers used in human motion analysis. Biomedicine 2003; 72: 155-165.

19. Cereatti A, Croce U, Cappozzo A. Reconstruction of skeletal movement using skin markers: comparative assessment of bone pose estimators. Journal of NeuroEngineering and Rehabilitation 2006, 3:7.

20. Rueterboriesa J, Spaicha E, Larsenb B, Andersena O. Methods for gait event detection and analysis in ambulatory systems. Medical Engineering \& Physics 2010; 32: 545-552.

21. Uluçam E, Sabri B. Measurement of Normal Lumbar Spine Range of Motion in the College-Aged Turkish Population Using a 3D Ultrasound-Based Motion Analysis System. Trakya Üniversitesi Tip Fakültesi Dergisi. 2009; Cilt 26, Sayl 1, Sayfa (lar) 029-035.

22. Tik-Pui D, Hong Y, Li J. Lower extremity preventive measures for slips - joint moments and myoelectric analysis. Ergonomics 2008; Vol. 51, No. 12; 1830-1846.

23. Bazański T. Original paper Metacarpophalangeal joint kinematics during a grip of everyday objects using the three-dimensional motion analysis system. Acta of Bioengineering and Biomechanics 2010; Vol. 12, No. 2.

24. Wolf A, Senesh M. Estimating joint kinematics from skin motion observation: modelling and validation. Computer Methods in Biomechanics and Biomedical Engineering First article, 2011, 1-8.

25. Barton C, Levinger P, Crossley K, Webster K, Menz H. Relationships between the Foot Posture Index and foot kinematics during gait in individuals with and without patellofemoral pain syndrome. Journal of Foot and Ankle Research 2011; 4:10.

26. Levinger P, Gilleard W. Relationship Between Static Posture and Rearfoot Motion During Walking in Patellofemoral Pain Syndrome Effect of a Reference Posture for Gait Analysis. J Am Podiatr Med Assoc 2006; 4: 323-329.

27. Chuter V. Relationships between foot type and dynamic rearfoot frontal plane motion. Chuter Journal of Foot and Ankle Research 2010, 3:9.

28. Stebbins J, Harrington M, Thompson N, Zavatsky A, Theologis T. Repeatability of a model for measuring multi-segment foot kinematics in children. Gait \& Posture 2006; 23:401-410. 2005.

29. Wright C, Arnold B, Coffey T, Pidco P. Repeatability of the modified Oxford foot model during gait in healthy adults. Gait \& Posture 2011; 33: 108-112.

30. Chiu H, Su F, Wang S, Hsu H. The motion analysis system and goniometry of the finger joints. Journal of Hand Surgery 1998; 23B: 788-791.

31. Ting H, Kuo L, Yi L, Wu W, Su F. The three-dimensional analysis of three thumb joints coordination in activities of daily living. Clinical Biomechanics 2011; 26: 371-376.

32. Shorter A, Polk, Rosengren K, Hsiao-Wecksler E. A new approach to detecting asymmetries in gait. Clinical Biomechanics 2008; 23:459467.

33. Cappozzo A, Croce U, Leardini A Chiari L. Human movement analysis using stereophotogrammetry Part 1: theoretical background. Gait and Posture 2005; 21: 186-196.

34. Chiari L, Croce U, Leardini A, Cappozzo A. Human movement analysis using stereophotogrammetry Part 2: Instrumental errors. Gait and Posture 2005; 21: 197-21.

35. Gil A, Pérez S, Forner A, Pérez E, Crespo B, Del Ama A. Gait kinematic analysis in patients with a mild form of central cord syndrome. Journal of Neuro Engineering and Rehabilitation 2011; 8:7.

36. Carson M, Harrington M, Thompson N, O'Connor J, Theologisc T. Kinematic analysis of a multi-segment foot model for research and clinical applications: a repeatability analysis. Journal of Biomechanics 2001; 34:1299-1307.

37. Groote F, Laet T, Jonkers I, Schutter J. Kalman smoothing improves the estimation of joint kinematics and kinetics in marker-based human gait analysis. Journal of Biomechanics 2008; 41: 3390-3398

38. Davis III R, Ounpuu S, Tyburski D, Gage J. A gait analysis data collection and reduction technique. Human movement science 1991; 10:575587.

39. Tinetti ME, Williams TF, Mayewski R, Fall Risk Index for elderly patients based on number of chronic disabilities. Am J Med 1986:80:429434.

40. Dutton M. Manual therapy of the spine an integrated approach. España. McGraw-Hill 2002.

41. Álvarez M, Benítez M, Espinosa J, Gorroñogoitia A, Muñoz F, Regato P. Atención A Las Personas Mayores desde la Atención Primaria. Barcelona. Semfyc. 